\title{
Polyaddition of Dithiol Compounds to Diethynyl Compounds: Kinetics of the Model Addition Reaction of Thiophenol to Ethynylbenzene
}

\author{
Eiichi Kobayashi,* Takatsugu Obata, Sadahito Aoshima, \\ and Junji Furukawa \\ Department of Industrial Chemistry, Faculty of Science and Technology, \\ Science University of Tokyo, Noda, Chiba 278, Japan
}

(Received May 12, 1990)

\begin{abstract}
The addition reaction mechanism of thiophenol to ethynylbenzene was studied as a model of the polyaddition of 1,4-benzenedithiol to 1,4-diethynylbenzene. The addition reaction occurred without appreciable side reactions and an adduct of the anti-Markownikoff's structure $(\phi \mathrm{SCH}=\mathrm{CH} \phi)$ was quantitatively obtained. It was found that the addition reaction is very similar to the polyaddition of 1,4-benzenedithiol to 1,4-diethynylbenzene from the point of the elementary reaction. The kinetic study shows that the rate-determining step of the addition reaction is the addition reaction of the thiyl radical to the ethynyl bond. The results are compared with those of the addition reaction of thiophenol to styrene.
\end{abstract}

KEY WORDS Addition Reaction / Reaction Mechanism / Kinetics / Reaction Order / Rate-Determining Step / Thiophenol / Ethynylbenzene / 1,4-Benzenedithiol / 1,4-Diethynylbenzene / Polyaddition /

Thiols have been known to add readily to acetylenes as well as olefins in the presence of radical initiators or light. ${ }^{1,2}$ In many cases the addition reactions of thiols to acetylenes proceed through two steps to give mono- and di-adducts of thiols. ${ }^{2}$ However, polymerizations by use of this sort of the addition reaction are scarcely known. Nuyken et al. reported the polyaddition of 1,3-benzenedithiol to 1,4diethynylbenzene (DEB) $)^{3,4}$ or alkyldiyne ${ }^{4}$ in 1988.

In this laboratory, the polyaddition of conjugated dithiol compounds to conjugated diethynyl compounds has been investigated since $1984 .{ }^{5}$ For example, the polyaddition of 1,4-benzenedithiol (BDT) to DEB takes place readily in solution or in solid state under UV irradiation or in the presence of radical initiators. A conjugated linear polymer of the
anti-Markownikoff's structure is obtained as eq $1 .^{6}$

$$
\begin{aligned}
& \mathrm{HC} \equiv \mathrm{C}-\langle\mathrm{O}\rangle \mathrm{C} \equiv \mathrm{CH}+\mathrm{HS}\langle\mathrm{O}\rangle \mathrm{SH} \stackrel{\dot{\mathrm{R}} \text { or } h v}{\longrightarrow} \\
& \mathrm{H} \mathrm{CH}=\mathrm{CH}-\mathrm{O} \text { - } \mathrm{CH}=\mathrm{CH}-\mathrm{S}-(\mathrm{O}) \mathrm{S} \mathrm{t}_{\mathrm{n}}
\end{aligned}
$$

The polymer has thermal resistance $\left(T_{\mathrm{d}} \simeq\right.$ $500^{\circ} \mathrm{C}$ in air) and exhibits relatively a large conductivity as $10^{-2} \mathrm{~S} \mathrm{~cm}^{-1}$ on doping with $\mathrm{I}_{2}{ }^{6}$ The polymer films obtained in solid state polymerization possess a layer structure by X-ray analysis. ${ }^{7}$ The amount of the layer structure of the polymer film increases or decreases reversibly by irradiation with light of particular wave lengths. ${ }^{8,9}$ Instead of benzene ring polymers containing various aromatic rings, ${ }^{10-12}$ hetero atoms ${ }^{13}$ in the main chain have been synthesized. However, the conjugated polymers did not dissolve in conventional organic solvents and the polym-

\footnotetext{
* To whom all correspondence should be addressed.
} 
erization mechanism is also unclear. In the present paper, the polymerization mechanism and polymer structure are investigated with the aid of the addition reaction of thiophenol to ethynylbenzene. The addition reaction mechanism was studied mainly by a kinetic method. The results were compared with the results of the addition reaction of thiophenol to styrene described in the previous paper. ${ }^{14}$

\section{EXPERIMENTAL}

\section{Reagents}

Thiophenol (Sumitomo Seika Chem. Co.) and ethynylbenzene (Wako Pure Chem. Co.) were obtained commercially. Both monomers were purified three times by vacuum distillation under nitrogen atmosphere before use. Their purities were confirmed to be over $99 \%$ by GC. Benzene (Wako Pure Chem. Co.) as a reaction solvent was distilled over $\mathrm{CaH}_{2}$ and then deaired to exclude oxygen by vacuum for $20 \mathrm{~min}$ before use. 2,2'-Azobisisobutyronitrile (AIBN; Wako Pure Chem. Co.) was used as received. Nitrogen gas was purified as shown in the previous paper ${ }^{14}$ and then through a glass column containing molecular sieve $3 \mathrm{~A}$ at near liquid nitrogen temperature, since the molecular sieve $3 \mathrm{~A}$ absorbs oxygen at low temperature. $^{15}$

\section{Addition Reaction}

In a two necked flask with a three-way stopcock and a W-cap, each benzene solution of ethynylbenzene $\left(0.3 \mathrm{moll}^{-1}\right)$, thiophenol $\left(0.3 \mathrm{moll}^{-1}\right)$, and AIBN $\left(3 \times 10^{-3} \mathrm{moll}^{-1}\right)$ was prepared using a syphon method under nitrogen atmosphere. The reaction was carried out by introducing each solution into a reaction vessel by the syphon method. The reaction temperature was maintained at $25^{\circ} \mathrm{C}$. The course of the reaction was monitored every $c a$. 10 min with measurement of the concentrations of both monomers by GC using decane as the internal reference.

\section{Measurements}

${ }^{1} \mathrm{H}$ NMR spectrum was obtained with a JEOL PMX-60 si in $\mathrm{CDCl}_{3}$ with tetramethylsilane as the internal standard. IR spectrum was recorded on a Hitachi 260-50 spectrophotometer by a liquid film method. FD mass spectra were obtained on a JEOL JMSMX110. The MWD curve was measured by a gel permeation chromatography (GPC) using a Tosoh CCPD dual pump, liquid chromatograph (Tosoh Tsk gel G2000HXL, G3000HXL, and $\mathrm{G} 4000 \mathrm{HXL}$ polystyrene gel columns, eluent $\mathrm{CHCl}_{3}$ ), and the molecular weight of the products was calculated on the basis of polystyrene. Gas chromatograms were measured on a Shimadzu GC-4BMPF equipped with a flame ionization detector (FID) and a silicon GE SE-30 column $(2 \mathrm{~m})$ at $140^{\circ} \mathrm{C}$ under nitrogen carrier gas.

\section{RESULTS AND DISCUSSION}

\section{Addition Reaction of Thiophenol to Ethy- nylbenzene}

Preparation of Addition Product of Thiophenol to Ethynylbenzene. Previous work ${ }^{14}$ has shown that a linear high polymer was formed in the polyaddition of BDT to 1,4-divinylbenzene and the model addition reaction of thiophenol to styrene took place quantitatively without a side reaction. Similarly, in order to know the reason why the polyaddition of BDT to DEB occurs preferentially, the addition reaction of thiophenol to ethynylbenzene was investigated in detail.

Thiophenol was reacted with an equimolar amount of ethynylbenzene in the presence of $2,2^{\prime}$-azobisisobutyronitrile (AIBN) as a radical initiator in benzene at $60^{\circ} \mathrm{C}$ under nitrogen atmosphere. The reaction was carried out under nitrogen atmosphere to avoid contamination by oxygen. ${ }^{14}$ There is no induction period in the reaction and both monomers are almost consumed in $2 \mathrm{~h}$. The crude product obtained by removal of the benzene from the reaction mixture without any pretreatment was 
a colorless transparent liquid. However, when the reaction was carried out in air, the reaction occurred vigorously on increasing the reaction temperature, and the product was a yellow liquid.

The crude product obtained under nitrogen atmosphere was analyzed by GPC and GC. About $99 \%$ of the product was found to be the $1: 1$ adduct of thiophenol to ethynylbenzene. The crude product contained $c a .0 .7$ $\mathrm{wt} \%$ and $0.3 \mathrm{wt} \%$ of oligomers of which molecular weights were 310 and 420, respectively. But, no by-products such as teromers of ethynylbenzene with higher molecular weights than the oligomers were confirmed (Figure 1). The crude product also contained $c a .0 .3 \mathrm{wt} \%$ of diphenyldisulfide formed by a coupling reaction of benzenethiyl radicals.

The structures of the products were analyzed by ${ }^{1} \mathrm{H}$ NMR and FD mass. The ${ }^{1} \mathrm{H}$ NMR spectrum of the $1: 1$ adduct purified by vacuum distillation (bp $122-123^{\circ} \mathrm{C} / 0.07 \mathrm{mmHg}$ ) shows signals at $\delta 6.2-6.8 \mathrm{ppm}$ due to vinylene protons $\left[2 \mathrm{H} ; \quad J_{\text {cis }}=10.5 \mathrm{~Hz}, J_{\text {trans }}=15.5 \mathrm{~Hz}\right.$ $\left.\left(J_{\text {cis }}=10.0 \mathrm{~Hz}, \quad J_{\text {trans }}=17 \mathrm{~Hz}^{16}\right)\right]$ and at $\delta$ $6.8-7.4 \mathrm{ppm}$ due to benzene ring protons $(10 \mathrm{H})$ as shown in Figure 2. The spectrum indicates the anti-Markownikoff's structure of the adduct. The structure was also confirmed by $\mathrm{CH}=\mathrm{CH}$ absorptions at 1350 and $940 \mathrm{~cm}^{-1}$ in the IR spectrum. These spectra resemble those of the polymer of BDT and DEB. ${ }^{6}$ The oligomers are estimated to be the $1: 2$ and $2: 2$ adducts of thiophenol and ethynylbenzene, because their molecular weights were found to be 314 and 424, respectively from the molecular ion peak in the FD mass spectrum. The $1: 2$ adduct of thiophenol and ethynylbenzene is considered to be structure 1 . The $2: 2$ adduct 2 is estimated to be formed by thiophenol adding again to the vinylene groups in $1: 2$ adduct 1 . The vinylene group of position one in $\mathbf{1}$ may be more reactive than that of position three due to the steric hindrance. 3 is not confirmed and 4 is considered to be hardly formed sterically. Accordingly, the structure of

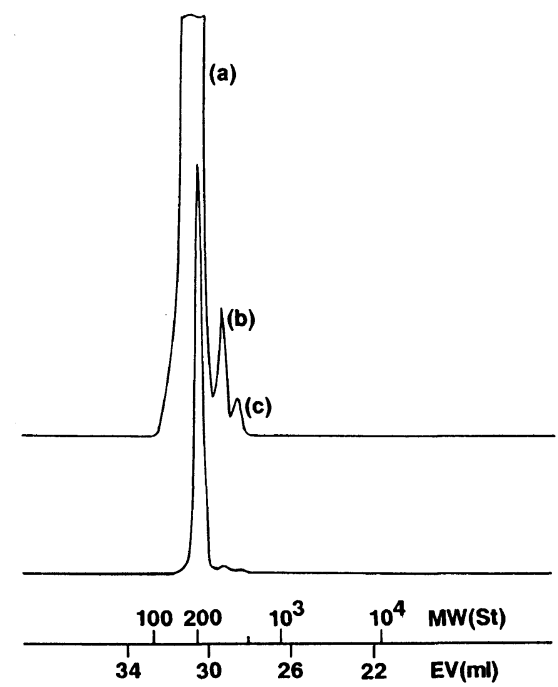

Figure 1. MWD curve of products obtained by the reaction of thiophenol and ethynylbenzene at $60^{\circ} \mathrm{C}$ for $2 \mathrm{~h}$ under nitrogen atmosphere: $[\phi \mathrm{SH}]_{0}=[\phi \mathrm{C} \equiv \mathrm{CH}]_{0}=$ $1 \mathrm{~mol} \mathrm{l}^{-1} ;[\mathrm{AIBN}]_{0}=0.01 \mathrm{moll}^{-1}$ in benzene; $\mathrm{a}, 210 ; \mathrm{b}$, $310 ; \mathrm{c}, 430$.

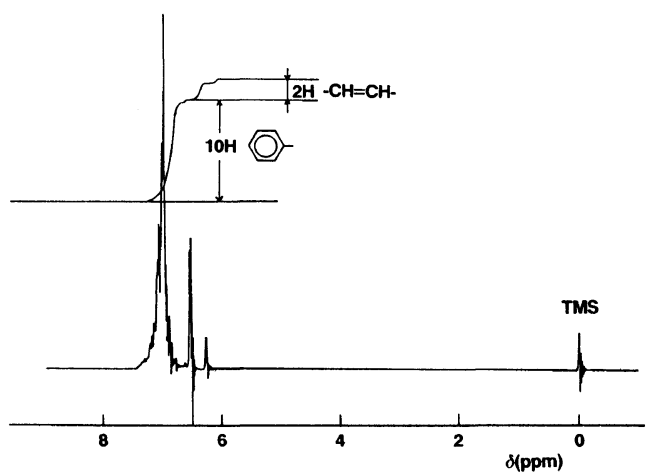

Figure 2. ${ }^{1} \mathrm{H}$ NMR spectrum of the adduct of thiophenol to ethynylbenzene in $\mathrm{CDCl}_{3}$.

$2: 2$ adduct is considered to be structure 2 . EI mass spectra may give information on the real structure of 2 or 4 .

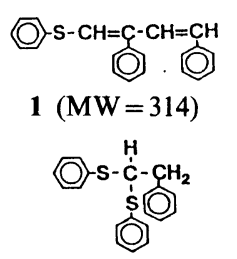

$3 \quad(\mathrm{MW}=322)$

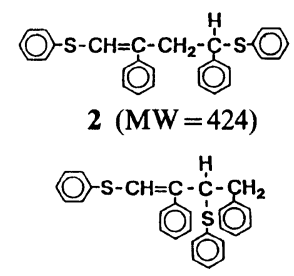

$4(\mathrm{MW}=424)$ 
It was indicated that the addition reaction of the anti-Markownikoff-type preferentially occurred without appreciable side reactions. Consequently, this addition reaction may be regarded as a model reaction of the polyaddition of BDT to DEB.

Reaction Mechanism. In the addition reactions of thiol compounds to ethynyl compounds, the stereochemistry has been studied in detail, ${ }^{16}$ but kinetic investigation has been done only scarcely. The addition reaction of thiophenol to ethynylbenzene is considered to progress by a chain mechanism similar to the addition reaction of thiophenol to styrene. ${ }^{14,17}$ Accordingly, the elementary reactions are as follows:

Initiation reaction

$$
\begin{array}{r}
\operatorname{AIBN} \stackrel{k_{\mathrm{d}}}{\longrightarrow} 2 \dot{\mathrm{R}}+\mathrm{N}_{2} \\
\dot{\mathrm{R}}+\phi \mathrm{SH} \stackrel{k_{\mathrm{i}}}{\longrightarrow} \phi \dot{\mathrm{S}}+\mathrm{RH}
\end{array}
$$

Addition reaction

Dissociation reaction

$$
\phi \dot{\mathrm{S}}+\phi \mathrm{C} \equiv \mathrm{CH} \underset{k_{\text {diss }}}{\stackrel{k_{\mathrm{a}}}{\rightleftharpoons}} \phi \mathrm{SCH}=\dot{\mathrm{C}} \phi
$$

Chain transfer reaction

$$
\dot{\mathbf{C}}+\phi \mathrm{SH} \stackrel{k_{\mathrm{tr}}}{\longrightarrow} \phi \mathrm{SCH}=\mathrm{CH} \phi+\phi \dot{\mathrm{S}}
$$

$\mathbf{A}$

Termination reaction

$$
\begin{array}{r}
2 \phi \dot{\mathrm{S}} \stackrel{k_{4}}{\longrightarrow} \mathrm{T}_{4} \\
\dot{\mathrm{C}}+\phi \dot{\mathrm{S}} \stackrel{k_{5}}{\longrightarrow} \mathrm{T}_{5} \\
2 \dot{\mathrm{C}} \stackrel{k_{6}}{\longrightarrow} \mathrm{T}_{6}
\end{array}
$$

Polymerization reaction

$$
\dot{\mathrm{C}}+\phi \mathrm{C} \equiv \mathrm{CH} \stackrel{k_{\mathrm{p}}}{\longrightarrow} \dot{\mathrm{P}}
$$

Di-addition reaction

$$
\mathbf{A}+\phi \dot{\mathbf{S}} \stackrel{k_{\mathrm{s}}}{\longrightarrow} \dot{\mathrm{D}}
$$

where $k_{\mathrm{d}}, k_{\mathrm{i}}, \cdots, k_{\mathrm{s}}$ are reaction rate constants of each elementary reaction. The dissociation reaction of $\beta$-phenylthiostyrylene radical, $\dot{\mathbf{C}}$, to ethynylbenzene and benzenethiyl radical may be negligible because of the resonance stabilization of $\dot{\mathbf{C}}$. The rate equation is given below under a steady state condition, since the addition reaction preferentially progresses without appreciable side reactions as mentioned above (see Figure 1),

$$
\frac{\mathrm{d}[\mathrm{A}]}{\mathrm{d} t}=\frac{\left(\frac{k_{\mathrm{d}}[\mathrm{AIBN}]}{k_{4}}\right)^{0.5} k_{\mathrm{tr}}[\phi \mathrm{SH}]}{\left[\frac{k_{\mathrm{tr}}[\phi \mathrm{SH}]}{k_{\mathrm{a}}[\phi \mathrm{C} \equiv \mathrm{CH}]}+\left(\frac{k_{6}}{k_{4}}\right)^{0.5}\right]}
$$

where $\mathrm{d}[\mathrm{A}] / \mathrm{d} t$ means the rate of the adduct formation. When the rate of the addition reaction $\left(k_{\mathrm{a}}\right)$ is much slower than that of the chain transfer reaction $\left(k_{\mathrm{tr}}\right)$, eq 8 is simplified as eq 9 ,

$$
\frac{\mathrm{d}[\mathrm{A}]}{\mathrm{d} t}=w k_{\mathrm{a}}[\phi \mathrm{C} \equiv \mathrm{CH}]^{1}[\mathrm{AIBN}]^{0.5}
$$

where $w$ is $\left(k_{\mathrm{d}} / k_{4}\right)^{0.5}$. On the other hand, assuming that the chain transfer reaction is the rate-determining step, the eq 8 is simplified as eq 10 ,

$$
\frac{\mathrm{d}[\mathrm{A}]}{\mathrm{d} t}=w^{\prime} k_{\mathrm{tr}}[\phi \mathrm{SH}]^{1}[\mathrm{AIBN}]^{0.5}
$$

where $w^{\prime}$ is $\left(k_{\mathrm{d}} / k_{6}\right)^{0.5}$. It is, therefore, realized that the rate-determining step in the addition reaction is able to be determined by the reaction order of both monomers.

Reaction Order and Rate-Determining Step. The reaction order of the ethynylbenzene was determined first. The reaction was carried out by changing the initial concentrations of ethynylbenzene, while the initial concentrations of thiophenol and AIBN were kept constant. Figure 3(a) shows the time-conversion curves of the adduct formation. The yield of the adduct of thiophenol and ethynylbenzene increases with reaction time and with increasing initial concentration of ethynylbenzene. Each 

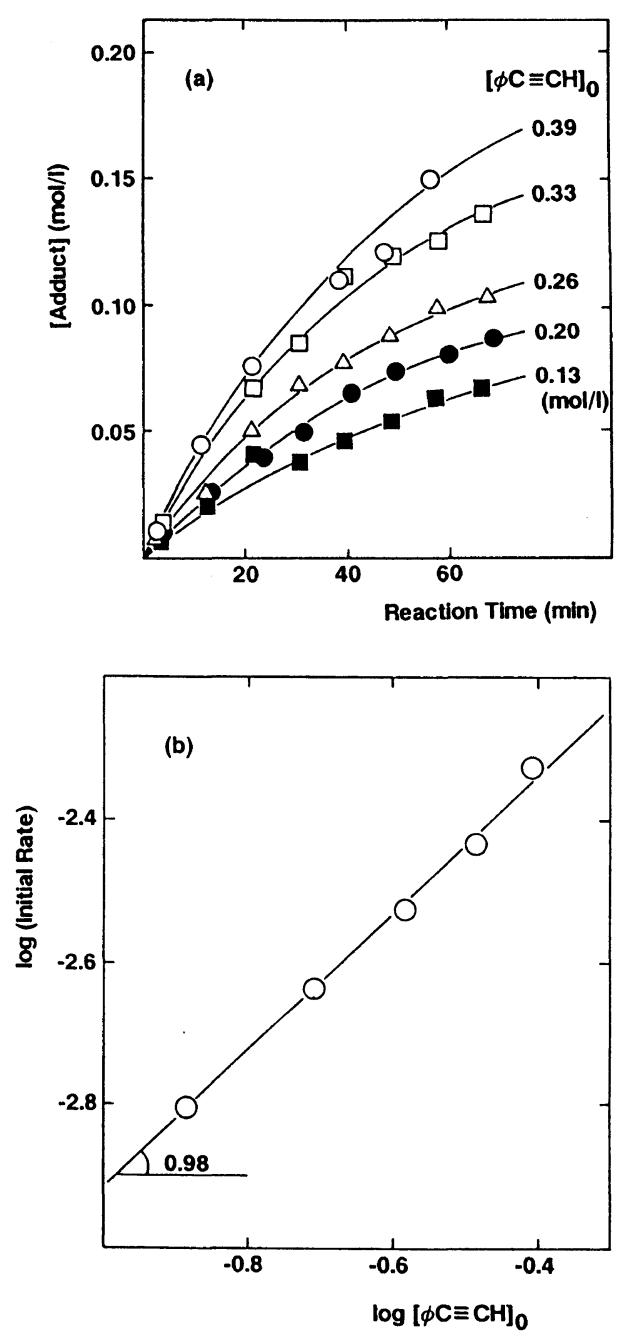

Figure 3. Time-conversion curves (a) of adduct formation by changing the initial concentration of ethynylbenzene and reaction order (b) of ethynylbenzene at $25^{\circ} \mathrm{C}$ in the dark under nitrogen atmosphere: $[\phi \mathrm{SH}]_{0}=$ $0.29 \mathrm{~mol} \mathrm{l}^{-1} ; \quad[\mathrm{AIBN}]_{0}=2.7 \times 10^{-3} \mathrm{~mol} \mathrm{l}^{-1}$ in benzene; internal ref. for GC, decane.

experimental point lays on a straight line drown by a pseudo first order assumption. The initial rates thus obtained are plotted against ethynylbenzene concentration (Figure 3(b)). The slope constant is about 1.0 indicating first order kinetics with respect to ethynylbenzene concentration. However, the rate of adduct formation is not affected by thiophenol con-
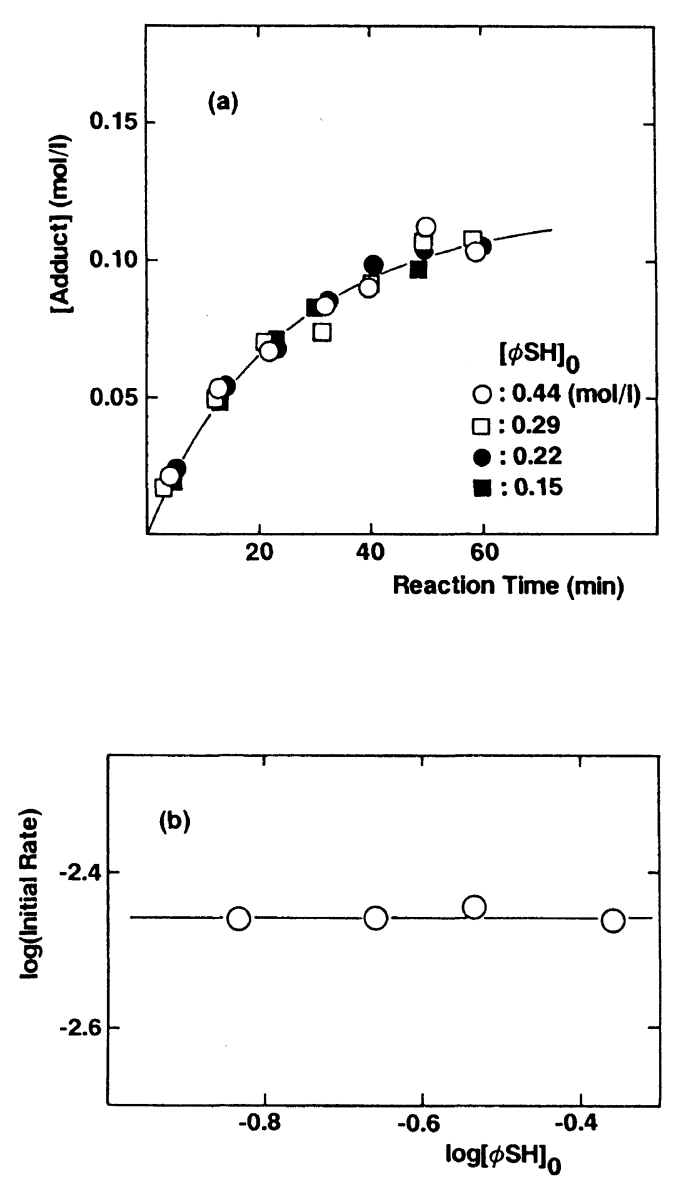

Figure 4. Time-conversion curves (a) of adduct formation by changing the initial concentration of thiophenol and reaction order (b) of thiophenol at $25^{\circ} \mathrm{C}$ in the dark under nitrogen atmosphere: $[\phi \mathrm{C} \equiv \mathrm{CH}]_{0}=0.29 \mathrm{~mol} \mathrm{l}^{-1}$; $[\mathrm{AIBN}]_{0}=3.1 \times 10^{-3} \mathrm{~mol}^{-1}$ in benzene; internal ref. for $\mathrm{GC}$, decane.

centration (Figure 4(a) and (b)). The kinetic order of AIBN is illustrated in Figure 5. The slope constant is approximately 0.5 .

This reaction may thus be expressed as the first order of ethynylbenzene, 0.5 order of AIBN and independent of the thiophenol concentration. The rate equation is as follows,

$$
\text { Rate }=k[\phi \mathrm{C} \equiv \mathrm{CH}]^{1}[\phi \mathrm{SH}]^{0}[\mathrm{AIBN}]^{0.5}
$$

where $k$ is the reaction rate constant in benzene at $25^{\circ} \mathrm{C}$. This experimental equation agrees with the case of eq 9 . This means that the 

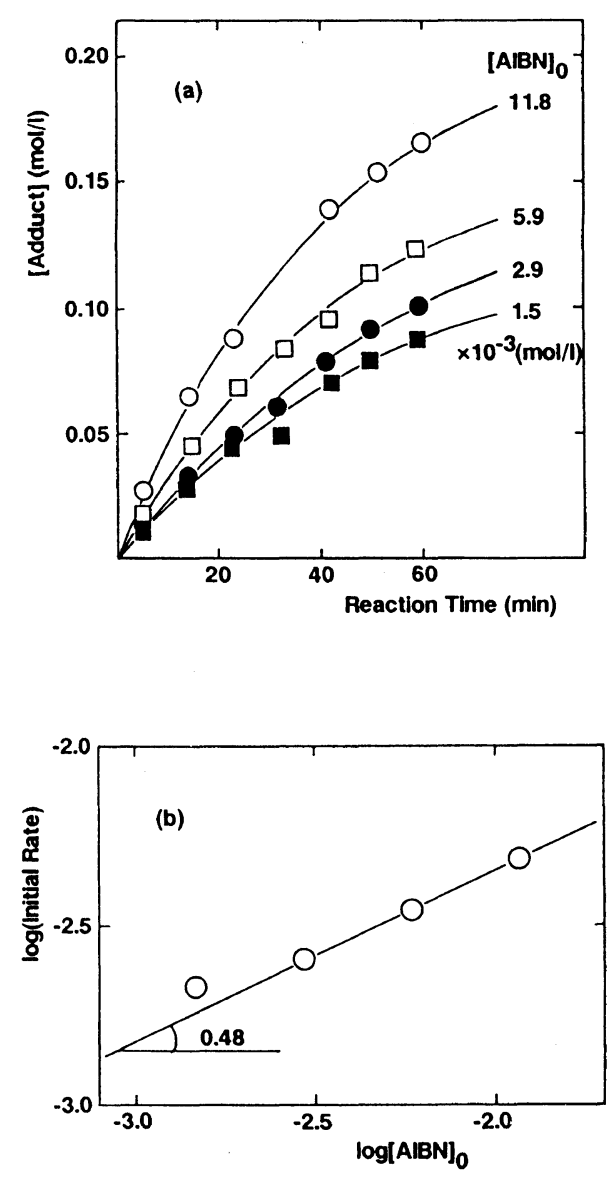

Figure 5. Time-conversion curves (a) of adduct formation by changing the initial concentration of AIBN and reaction order (b) of AIBN at $25^{\circ} \mathrm{C}$ in the dark under nitrogen atmosphere: $[\phi \mathrm{C} \equiv \mathrm{CH}]_{0}=0.29 \mathrm{~mol} \mathrm{1}^{-1}$; $[\phi \mathrm{SH}]_{0}=0.31 \mathrm{~mol} \mathrm{1}^{-1}$ in benzene; internal ref. for GC, decane.

addition reaction of the benzenethiyl radical to ethynylbenzene is the rate-determining step (eq 3). The result agrees with the result by molecular orbital calculations of the addition reaction of thiophenol to ethynylbenzene. ${ }^{18}$

According to the reaction scheme of the model adduct formation, the authors propose the polyaddition mechanism. In the case of the polyaddition of BDT to DEB, polymerization proceeds rapidly under UV irradiation in toluene at $-20^{\circ} \mathrm{C}$ and the polymer is obtained in a high yield. Two ethynyl groups are exclusively assembled in the polymer backbone and there is no pendant ethynyl group except polymer ends. That is, the polymer as well as the adduct have the anti-Markownikoff's structure. The cross-linked polymer may be scarcely obtained in the polyaddition, because the polymer of a molecular weight of about $2 \times 10^{4}$ may have potentially only one pendant ethynyl group according to the calculation based on experimental data obtained in the adduct preparation. Similarly, the absorption of the $\mathrm{S}-\mathrm{S}$ linkage in the IR spectra of the polymer is not confirmed. Only one S-S bond formation by a homopolymerization of BDT may be contained in the polymer of the molecular weight of about $5 \times 10^{4}$ by similar calculation. The molecular weight of the polymer, however, is $7.5 \times$ $10^{3}$ and a relative low value. ${ }^{6}$ Consequently, the insolubleness of the polymer of BDT and DEB in conventional organic solvents may be caused by a molecular aggregation owing to delocalized $\pi$ electrons along the polymer chain but not by a crosslinkage.

\section{Comparison between Styrene and Ethynylben- zene}

Relative Reactivity to Thiophenol. The differences in reactivities between ethynyl and vinyl groups have been investigated in bromination $^{19,20}$ and hydroboration. ${ }^{21}$ The results indicate that the reactivities of the vinyl compounds were much higher than those of ethynyl compounds. But, the relative reactivity of the radical addition reaction of thiol compounds to ethynyl and vinyl compounds has scarcely been studied. Sulimov et al. ${ }^{22}$ only reported the addition reaction of thiophenol to vinylacetylene.

$$
\begin{gathered}
\mathrm{HC} \equiv \mathrm{C}-\mathrm{CH}=\mathrm{CH}_{2}+\phi \mathrm{SH} \stackrel{(t-\mathrm{BuO})_{2}}{\longrightarrow} \\
\mathrm{HC} \equiv \mathrm{C}-\mathrm{CH}_{2} \mathrm{CH}_{2} \mathrm{~S} \phi
\end{gathered}
$$

In the reaction, the thiophenol did not add to the ethynyl group but to the vinyl group of vinylacetylene. This indicates that the reactivity of the vinyl group is higher than that of the 


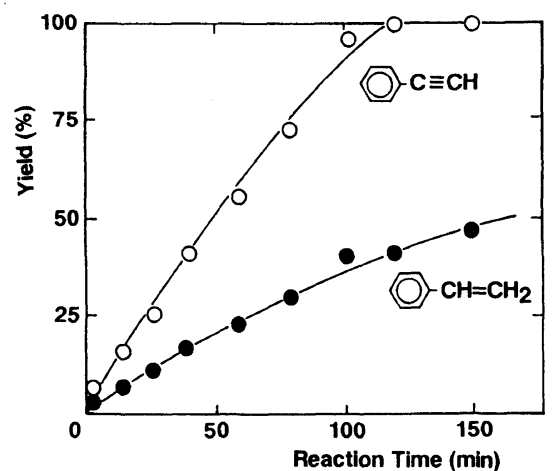

Figure 6. Competitive addition reaction of thiophenol to styrene and ethynylbenzene at $40^{\circ} \mathrm{C}$ in the dark under nitrogen atmosphere: $\left[\phi \mathrm{CH}=\mathrm{CH}_{2}\right]_{0}=[\phi \mathrm{C} \equiv \mathrm{CH}]_{0}=$ $0.16 \mathrm{moll}^{-1} ; \quad[\phi \mathrm{SH}]_{0}=0.31 \mathrm{moll}^{-1} ; \quad[\mathrm{AIBN}]_{0}=3.4 \times$ $10^{-3} \mathrm{~mol} \mathrm{l}^{-1}$ in benzene; internal ref. for GC, decane.

ethynyl group.

The authors also investigated differences in the reactivity between conjugated ethynyl and vinyl groups. The relative reactivity was determined by a competitive reaction of thiophenol to styrene and ethynylbenzene in benzene at $40^{\circ} \mathrm{C}$ under nitrogen atmosphere. Figure 6 shows the time-conversion curves of the adduct formation of thiophenol to styrene and ethynylbenzene. Thiophenol adds to ethynylbenzene more rapidly than styrene, and the addition reaction of thiophenol to ethynylbenzene is completed about $100 \mathrm{~min}$. Ethynylbenzene has three times as a high reactivity as styrene. This suggests that the rate of the addition reaction of benzenethiyl radical to ethynylbenzene is three times larger than the rate of the hydrogen abstraction from thiophenol by $\beta$-phenylthiostyryl radical $\left(\phi \mathrm{SCH}_{2} \dot{\mathrm{C}} \mathrm{H} \phi\right)$, because the rate-determining steps in both reactions are different as mentioned above. Such a high reactivity of ethynyl groups has been already expected from the polyaddition of BDT to DVB ${ }^{23}$ and BDT to DEB. ${ }^{6}$

In the case of the above-mentioned vinylacetylene, thiophenol adds preferentially to the vinyl group. HOMO and LUMO of acetylene are close to SOMO of thiyl radical than those of ethylene. Consequently, the reason
Table I. Comparison of addition reactions of thiophenol to styrene and ethynylbenzene

\begin{tabular}{ccc}
\hline & $\phi \mathrm{CH}=\mathrm{CH}_{2}$ & $\phi \mathrm{C} \equiv \mathrm{CH}$ \\
\hline Yield $/ \%$ & $\simeq 100$ & $\simeq 100$ \\
\hline Structure & $\begin{array}{c}\text { Anti- } \\
\text { Markownikoff }\end{array}$ & $\begin{array}{c}\text { Anti- } \\
\text { Markownikoff }\end{array}$ \\
\hline $\begin{array}{c}\text { Reaction order } \\
{[\mathrm{AIBN}]} \\
{\left[\phi \mathrm{CH}=\mathrm{CH}{ }_{2}\right] \text { or }} \\
{[\phi \mathrm{C} \equiv \mathrm{CH}]} \\
{[\phi \mathrm{SH}]}\end{array}$ & 0.5 & 0.5 \\
\hline $\begin{array}{c}\text { Rate-determining step } \\
\text { Chain transfer }\end{array}$ & Addition \\
\hline
\end{tabular}

for the high reactivity of vinyl group than ethynyl one in the eq 12 is unclear at the moment.

Comparison between Styrene and Ethynylbenzene in the Addition Reaction with Thiophenol. The results of the addition reaction of thiophenol to ethynylbenzene and to styrene $^{14}$ are summarized in Table I. Thiophenol adds quantitatively to both styrene and ethynylbenzene and side reactions scarcely occur. The adducts of the anti-Markownikoff's structure were obtained in nearly $100 \%$ yield. However, the reaction orders of the addition reactions of thiophenol to styrene were quite the opposite to those of ethynylbenzene. In the case of styrene, the rate-determining step is the chain transfer reaction between $\beta$-phenylthiostyryl radical $\left(\phi \mathrm{SCH}_{2} \dot{\mathrm{C}} \mathrm{H} \phi\right)$ and thiophenol. However, in the case of ethynylbenzene the addition reaction of the benzenethiyl radical to ethynylbenzene is the rate-determining step. That is, in the case of styrene the rate of the addition step, $R_{\mathrm{a}}^{\mathrm{St}}$, is extremely faster than the rate of the chain transfer step, $R_{\mathrm{tr}}^{\mathrm{St}}$ $\left(R_{\mathrm{a}}^{\mathrm{St}}>R_{\mathrm{tr}}^{\mathrm{St}}\right)$. On the contrary, in the case of ethynylbenzene the addition rate, $R_{\mathrm{a}}^{\mathrm{EB}}$, is much slower than the chain transfer rate, $R_{\mathrm{tr}}^{\mathrm{EB}}$ $\left(R_{\mathrm{a}}^{\mathrm{EB}}<R_{\mathrm{tr}}^{\mathrm{EB}}\right)$. The rate constants of the addition reaction of benzenethiyl radical to styrene and ethynylbenzene were determined to be $2 \times 10^{7}$ $\mathrm{M}^{-1} \mathrm{~s}^{-1}$ in cyclohexane ${ }^{24}$ and $7.9 \times 10^{5}$ 
$\mathrm{M}^{-1} \mathrm{~s}^{-1}$ in benzene ${ }^{25}$ at $23^{\circ} \mathrm{C}$ by the flash photolysis method, respectively. Accordingly, the rates of the addition step and chain transfer step in both cases of styrene and ethynylbenzene decrease in the following order; $R_{\mathrm{tr}}^{\mathrm{EB}} \geq R_{\mathrm{a}}^{\mathrm{St}}>R_{\mathrm{a}}^{\mathrm{EB}}>R_{\mathrm{tr}}^{\mathrm{St}}$. A perturbation of orbitals between the substrates such as the thiyl radical and styrene or ethynylbenzene may give a key for understanding the order of each elementary rate constant. This will be discussed in the next paper.

Acknowledgment. The authors are indebted to Mr. K. Tanaka (Idemitsu Kosan Co.) for taking the FD mass spectra.

\section{REFERENCES}

1. L. N. Owen and M. U. S. Sultanbawva, J. Chem. Soc., 3109 (1949).

2. H. Bader, L. C. Cross, I. Heilbron, and E. R. H. Jones, J. Chem. Soc., 619 (1949); K. Yamagishi, T. Tanaka, and T. Hoshino, Bull. Chem. Soc. Jpn., 30, 455 (1957); ibid., 29, 447 (1956); A. T. Blomquist and J. Walinsky, J. Org. Chem., 23, 5551 (1958).

3. O. Nuyken and F. Siebzehnrübl, Polym. Bull., 19, 371 (1988).

4. O. Nuyken and F. Siebzehnrübl, Makromol. Chem., 189, 541 (1986).

5. E. Kobayashi, T. Ohashi, and J. Furukawa, Polym. Prepr. Jpn., 33, 461 (1984).

6. E. Kobayashi, T. Ohashi, and J. Furukawa, Makromol. Chem., 187, 2525 (1986).

7. E. Kobayashi, T. Ohashi, and J. Furukawa, $J$. Polym. Sci., A, Polym. Chem., 25, 2077 (1987).
8. E. Kobayashi, T. Ohashi, T. Yagi, and J. Furukawa, Kasen Kouenshu, 44, 95 (1987).

9. E. Kobayashi, Kobunshi, 38, 836 (1989).

10. E. Kobayashi, Y. Ishizuka, Y. Kouchaku, and J. Furukawa, Kobunshi Ronbunshu, 46, 203 (1989).

11. E. Kobayashi, J. Jiang, H. Ohta, and J. Furukawa, J. Polym. Sci., A, Polym. Chem., 28, 2641 (1990); E. Kobayashi, J. Jiang, and J. Furukawa, Polym. J., 22, 266 (1990).

12. E. Kobayashi, S. Onda, T. Miyaji, S. Aoshima, and J. Furukawa, Polym. Prepr. Jpn., 38, 330 (1989).

13. E. Kobayashi, N. Metaka, H. Takayanagi, S. Aoshima, and J. Furukawa, Polym. Prepr. Jpn., 38, 1581 (1989).

14. E. Kobayashi, T. Obata, S. Aoshima, and J. Furukawa, Polym. J., 22, 803 (1990).

15. A. Tolk, W. Lingerak, A. Kout, and D. Börger, Anal. Chim. Acta, 45, 137 (1969).

16. A. A. Oswald, K. Griesbaum, B. E. Hudson, Jr., and J. H. Bregman, J. Am. Chem. Soc., 93, 3801 (1971).

17. R. H. Pallen and C. Sivertz, Can. J. Chem., 35, 723 (1957).

18. E. Kobayashi, H. Takayanagi, S. Aoshima, and J. Furukawa, Polym. Prepr. Jpn., 37, 1511 (1988).

19. G. H. Schmid, A. Modro, and K. Yates, J. Org. Chem., 42, 2021 (1977).

20. G. Modenas, F. Rivetti, and U. Tonellato, J. Org. Chem., 43, 1521 (1978).

21. C. A. Brown and R. A. Coleman, J. Org. Chem., 44, 2328 (1979).

22. I. G. Sulimov and A. A. Petrov, Zhur. Organ. Khim., 2, 767 (1966) [Chem. Abstr., 65, 12099 (1966)].

23. T. Yagi, G. Morishita, and E. Kobayashi, Zairyo Gijyutsu, 7, 272 (1989).

24. O. Ito and M. Matsuda, J. Am. Chem. Soc., 101, 5732 (1979).

25. O. Ito, R. Omori, and M. Matsuda, J. Am. Chem. Soc., 104, 3934 (1982). 